\title{
Mapping Thematic Trends and Analysing Hotspots Concerning the Use of Stem Cells for Cartilage Regeneration: a Bibliometric Analysis From 2010 to 2020
}

\section{Demeng Xia}

Naval Hospital of Eastern Theater

Jianghong Wu

Changhai Hospital

Kaiwen Wu

Chengdu Third People's Hospital

Sheng Wang

Changhai Hospital

\section{Zhentao Zhang}

Naval Medical University

Panyu Zhou

Changhai Hospital

Shuogui Xu ( $D$ 18516116672@163.com)

Changhai Hospital https://orcid.org/0000-0001-5926-4391

\section{Research}

Keywords: stem cells, cartilage regeneration, bibliometric analysis

Posted Date: February 23rd, 2021

DOI: https://doi.org/10.21203/rs.3.rs-230960/v1

License: (9) This work is licensed under a Creative Commons Attribution 4.0 International License. Read Full License

Version of Record: A version of this preprint was published at Frontiers in Pharmacology on January 3rd, 2022. See the published version at https://doi.org/10.3389/fphar.2021.737939. 


\section{Abstract}

Background: Defects of articular cartilage are a common condition that usually progresses to osteoarthritis with pain and dysfunction of the joint. Current treatment strategies have had limited success in these patients. Stem cells are emerging as a promising option for cartilage regeneration. We aim to summarize the developmental history of stem cells for cartilage regeneration and to analyse the trends and hotspots.

Methods: We screened all relevant literature on stem cells for cartilage regeneration from Web of Science during 2010-2020 and analysed the research trends in this field by VOSviewer and CiteSpace. We also summarized previous clinical trials.

Results: We screened 1,011 publications. China contributed the largest number of publications (317, $31.36 \%)$ and citations $(81,376,48.61 \%)$. The United States has the highest H-index (39). Shanghai Jiao Tong University had the largest number of publications (34) among all full-time institutions. The Journal of Biomaterials and Stem Cell Research \& Therapy published the largest number of studies on stem cells for cartilage regeneration (35). SEKIYA I and YANG F published the majority of articles in this field (14), while TOH WS was cited most frequently (740). Regarding clinical research on stem cells for cartilage regeneration, the keyword "double-blind" emerged in recent years, with an average year of 2018.75. In tissue engineering, the keyword "3D printing" appeared latest, with an average year of 2019.625. In biological studies, the key word "extracellular vesicles" appeared latest, with an average year of 2018.9091. The current research trend indicates that basic research is gradually transforming into tissue engineering. Clinical trials have confirmed the safety and feasibility of stem cells for cartilage regeneration.

Conclusions: Multiple scientific methods were employed to reveal productivity, collaborations, and research hotspots related to the use of stem cells for cartilage regeneration. 3D printing, extracellular vesicles, and double-blind clinical trials are research hotspots and are likely to be promising in the near future. Further studies are needed for to improve our understanding of this field, and clinical trials with larger sample sizes and longer follow-up periods are needed for clinical transformation.

\section{Introduction}

Articular cartilage (AC) is a layer of hyaline cartilage with unique viscoelastic characteristics. AC lubricates articulation and disperses the stress in the joint to protect it from defects. However, traumatic and degenerative injuries of $A C$ and untreated cartilage injuries eventually progress to osteoarthritis (OA), which is the most common type of musculoskeletal disorder ${ }^{1}$. Articular cartilage has very poor healing capability; because of its avascular nature, mature chondrocytes have a weak ability to proliferate and produce sufficient extracellular matrix (ECM) to fill a defect ${ }^{2}$. A large number of studies have been carried out to simulate cartilage regeneration. Surgical techniques to treat focal chondral defects include marrow stimulation (MST) surgery, such as the current first-line treatment microfracture (MF) surgery. Although 
the resulting 'fibrocartilage' provides some symptomatic relief, it has substantially weakened mechanical properties compared with those of normal articular cartilage ${ }^{3,4}$. In comparison, cell-based regenerative therapy can provide a long-term solution, alleviate symptoms and finally delay OA progression. Autologous chondrocyte transplantation (ACl) has been approved by the FDA and is currently in clinical practice. ACl needs to isolate mature chondrocytes and culture them in vitro, which inevitably has limitations such as limited cells, in vitro chondrocyte dedifferentiation, and donor-site morbidity caused by cartilage harvest. ${ }^{5}$ To overcome the limitations of the current methods, the potential of stem cells for cartilage regeneration has attracted widespread interest.

Human embryonic stem cells (hESCs) are considered to be a promising cell source for cartilage regeneration because of their unlimited self-renewal capacity and multidirectional differentiation potential ${ }^{6}$. As the field of stem cells continues to expand, more stem cell types have been discovered. Currently, the majority of cartilage regeneration research focuses on MSCs, which can be obtained from mesenchymal tissues and have the capacity to proliferate and differentiate into chondrocytes ${ }^{7,8}$. Clinical trials are systematic studies conducted in humans and are indispensable for determining efficacy and safety. The effectiveness of clinical trials is a prerequisite for further clinical applications. Clinical trials on the potential of stem cells in cartilage regeneration have also been carried out ${ }^{9}$. Some types of stem cells have already entered clinical trials, among which the hUCB-MSC-based therapeutic product CARTISTEM has been approved by the Korean FDA for marketing. Regenerative strategies for AC are the focus of study, and the scientific community (as well as researchers, research institutions, etc.) is constantly exploring this field; therefore, it is urgent to summarize and analyse the trends of this field.

The literature is the carrier of scientific progress. Bibliometric analysis uses mathematical and statistical methods to quantitatively and quantitatively analyse publications in medical databases, revealing the development history, research focus and future trends of a certain field. In addition, bibliometric analysis can implement comparisons between different countries or institutions by analysing the quality and quantity of previous research ${ }^{10,11}$. As much progress has been made concerning the potential of stem cells in cartilage regeneration, the number of related papers has also increased drastically, but related bibliometric studies have not been performed. In this study, we analysed publications about stem cells for cartilage regeneration in the Web of Science (WoS) database during 2010-2020. We conducted statistical analysis on the number of publications, keyword frequency, and citation frequency. We conducted qualitative and quantitative analyses of research from different countries, institutions, journals and authors. We also summarized previous clinical trials. We aim to map thematic trends and hotspots related to the capability of stem cells for cartilage regeneration, providing a reference for future research.

\section{Material And Methods}

\subsection{Data acquisition and search strategy}

Web of Science is an important academic database worldwide that has been widely used in bibliometric analysis. We selected WoS as a data source for a comprehensive search on stem cells for cartilage 
regeneration from 2010 to 2020 . The search strategies were as follows: TS=(stem cell* OR SC) and ( ${ }^{*}$ cartilage or chondro*) and regenera* AND language: (English). The screening process is shown in Figure 1. Relevant clinical trial data were obtained from ClinicalTrials.gov (https://clinicaltrials.gov/), the keywords were the same, and the limited condition was "completed study". All data were obtained online, and no ethical certification was required. All searches were conducted on November 27, 2020, to avoid bias related to database updates.

\subsection{Data collection}

Two authors (Demeng Xia and Jianghong Wu) independently screened and extracted all data from the final included articles, including titles, keywords, authors, institutions, journals, dates of publication, countries/regions, citations, H-index, etc. Then, we conducted analysis with Microsoft Excel 2016, CiteSpace version $\mathrm{V}$ and VOSviewer.

\subsection{Bibliometric analysis}

Relative research interest (RRI) is an indicator of activity in a certain field, and RRI is defined by weighted publications per year (WPPY) in a certain field and all weighted publications per year in PubMed (AWPPY). We used Excel to calculate the RRI and to draw the time curve. The impact factor (IF) was acquired in the newest edition of the journal citation report (JCR). The H-index is an evaluation index of the academic achievement of scientists or countries, and the H-index is defined by both productivity and citation impact per publication according to previous studies (scientists or countries have published " $\mathrm{H}$ " papers, and the papers have been cited in other publications at least " $\mathrm{H}$ " times). The visualization software programs VOSviewer and CiteSpace are widely used in bibliometric analysis. We employed VOSviewer software to perform coupling analysis of nations and institutions, co-citation analysis of journals, and co-occurrence of key words. The average appearing year (AAY) was used to describe the relative novelty of keywords. We employed CiteSpace mainly to perform co-citation analysis of references.

\section{Result}

\subsection{Field activity and global contributions}

\subsubsection{Field activity analysis}

Depending on the inclusion criteria, a total of 1011 articles related to stem cells for cartilage regeneration were included in the final analysis. According to the annual distribution of publications, although there was a slight decline in 2010-2011 (35-26 publications per year), 2014-2015 (65-46 publications per year) and 2018-2019 (166-146 publications per year), the overall trend of the publication output increased from 2010 to 2020 (34-271 publications per year). The trend of the RRI was similar, suggesting that the field of stem cells for cartilage regeneration is receiving more attention in general (figure $2 \mathrm{~A}$ ).

\subsubsection{Global contribution to the field}


According to the country distribution of publications, China (317 publications) is the most productive country, followed by the USA (203 publications), South Korea (107 publications), Japan (82 publications) and Germany (59 publications) (figure 2B). It is more intuitive to identify these publishing centres from the density map (figure 2C). The top 20 countries with the most publications are listed in Table 1.

In terms of total citations, the top 4 countries are China (5351 citations), the USA (5081 citations), South Korea (3048 citations) and Japan (1374 citations). Singapore is not in the top 10 productive countries (25 publications) but ranks fifth for total citations (1253 citations). The countries with the highest $\mathrm{H}$-index are as follows: first, the USA (39); second, China (37); third, South Korea (29); fourth, Japan (21); and fifth, Italy (18) (figure 2B).

The collaborations between countries are shown in Figure 2D. The size of the circles indicates the number of publications, and the width of the connecting line between two circles indicates the degree of collaboration. The USA has the strongest total link strength, which means that the United States has a strong influence in this field. Many countries have concentrated years of article output. France, Germany and Singapore had the most publications concentrated before 2016. Then, the U.S. and Japan published articles intensively in the next two years. Articles from England and mainland China were mainly published after 2018.

\subsubsection{Analysis of institution distribution}

The 4 institutions with the most publications are all located in China, and sequentially, they are Shanghai Jiao Tong University (34 publications), Peking University (27 publications), Sichuan University (26 publications), and Chinese PLA General Hospital (22 publications). The Chinese Academy of Science (21 publications) and National University of Singapore (21 publications) tied for $5^{\text {th }}$. The top 20 institutions with the most publications are listed in Table 2.

Figure $3 \mathrm{~A}$ demonstrates that there are close and complex collaborative relations between different institutions. CiteSpace was employed to analyse the centrality of institutions. The purple circle indicates centrality, and the area of the circle is proportional to the centrality. Shanghai Jiao Tong University and National University of Singapore are the most prominent institutions, which suggests that they are regarded as pivotal points (figure 3B).

\subsubsection{Analysis of journal distribution}

Different journals have different fields of publication. Therefore, we performed a journal distribution analysis of publications on stem cells for cartilage regeneration. The journals Biomaterials (impact factor $=10.317,2019$ ) and Stem Cell Research \& Therapy (impact factor= 5.116, 2019) published the most studies, both of which had 35 publications. There were 34 articles on stem cell research for cartilage regeneration in Stem Cells International (IF = 3.869, 2019), 33 articles in Acta Biomaterialia (IF $=7.242$, 2019) and 32 articles in Journal of Tissue Engineering Part $A(I F=3.776,2019)$. The top 20 journals with the most publications are listed in Figure 4A. 
The journals are not kept in isolation. Biomaterials and Acta Biomaterialia have the closest relationship. As far as this field is concerned, journals with a high volume of publications also have high centrality (figure 4B).

\subsection{0verview of landmark articles and authors}

The authors with the highest number of publications in 2010-2020 are shown in Table 3, and the total citations of the publications are also listed. SEKIYA I and YANG F tied for first place. However, TOH WS of the National University of Singapore is particularly noteworthy. Although TOH WS published only 10 articles, the total number of citations reached 740 , contributing greatly to Singapore's total citations. This shows that his viewpoint has drawn widespread attention in the field.

We analysed the references of the shortlisted publications and constructed the cocitation network. Jo Ch's article (doi:10.1002/stem.1634) published in Stem Cells in 2014 is at a key node in the co-citation network (figure 5A). We further performed an in-depth analysis of the article with the highest citation rate (Figure 5B). Then, we implemented a cluster analysis. The references in the co-citation network are divided into 12 diverse clusters (figure $5 \mathrm{C}$ ). The clusters are labelled by extricating terms from the titles of the cited publications. From a timeline perspective, the theoretical basis of earlier publications is mainly osteogenesis and growth factors. Articles in recent years have been based on hydrogels and mesenchymal stem cells. The top 10 articles in total citations are detailed in Table 4.

The burst detection function in CiteSpace is helpful to obtain the research frontiers. The top 25 strongest citation bursts of the references are presented, and there was no article with a sudden change in the number of citations in the past 3 years (figure 5D).

\subsection{Co-occurrence analysis of key words}

Keywords represent the main topic of publications. Co-occurrence analysis of keywords is conducive to systematically understanding the relationship between keywords and consequently grasping the relationship between various topics in this field. Further cluster analysis helps to systematically understand the current progress in this field. VOSviewer was employed to analyse keywords (defined as words that were used more than five times in titles and abstracts in all publications) in all included publications. A total of 203 identified keywords were mainly divided into three clusters: 'clinical study', 'tissue engineering' and 'biological study'. In clinical study, the keywords with the highest frequency were repair (273 times), articular cartilage (238 times), osteoarthritis (264 times), regeneration (222 times) and bone marrow (193 times). In tissue engineering, these words were chondrogenic differentiation (203 times), scaffold* (160 times), mesenchymal stem cells (152 times), tissue engineering (108 times) and bone (97 times). In biological study, the words were differentiation (282 times), chondrogenesis (208 times), cartilage (196 times), tissue (191 times) and chondrocytes (186 times) (figure 6A, figure 6B). The annual distribution of keywords is indicated. Chronological order is presented from dark blue to bright yellow. In "Clinical study", the earliest keyword was "autologous chondrocyte transplantation" (AAY 2014.7143), with 14 times, and the latest word was "double-blind" (AAY 2018.75), with 8 times. In tissue 
engineering, the earliest word was "growth factors" (AAY 2014.125), with 8 times, and the latest word was "3d printing" (AAY 2019.625), with 9 times. In the biological study, the earliest word was "cartilage formation" (AAY 2014.875), with 8 times, and the latest word was "extracellular vesicles" (AAY 2018.9091), with 12 times (figure 6C). According to the colour intensity, we found that the research hotspot on stem cells for cartilage regeneration gradually shifted to tissue engineering in recent years (Table S1). The 20 keywords with the strongest citation burst are listed in Figure 6D, and no keywords had a sudden change in the number of citations in the past 3 years.

\subsection{Clinical research summary}

Clinica/Trials.gov (https://clinicaltrials.gov/) is one of the most important databases of clinical studies conducted around the world, and it was developed by the National Library of Medicine (NML) and the U.S. Food and Drug Administration (FDA). ClinicalTrials could provide inquiry services of clinical trial information to the general public and could also provide clinical trial registration services to medical researchers and institutions. We used ClinicalTrials.gov to list the currently completed clinical studies on stem cells for cartilage regeneration (table 5). A total of 16 clinical trials have been completed, and among them, CARTISTEM has been approved by the Korean FDA. The 16 clinical trials came from 12 countries, with the top 3 countries being Iran (3), Republic of Korea (2) and Spain (2). Less than one-third of clinical trials have a comparison group, and more than two-thirds have not yet entered Phase 3 . From the perspective of the size of the included samples, the overall size is small. Only NCT01041001 and NCT01626677 exceed 100, both of which are from the Republic of Korea and relevant to CARTISTEM. NCT01041001 assesses and compares the safety and efficacy of the allogeneic-unrelated umbilical cord blood-derived mesenchymal stem cell product (CARTISTEM) to that of a microfracture treatment, and NCT01626677 is a long-term follow-up study to investigate its safety and efficacy. In addition, the most important condition of these clinical trials is knee-related cartilage regeneration, which may be related to its high incidence and high feasibility for performing clinical research. From the perspective of article publication, only 6 projects have obtained results.

\section{Discussion}

This study offered a bibliometric analysis of stem cells for cartilage regeneration from 2010 to 2020 . With the development of bibliometric software, bibliometric analysis is now widely used. It can help beginners to understand the development process and trends of a specific field intuitively and systematically. It is also beneficial to find milestone achievements and new research hotspots.

Regenerative therapy uses multidisciplinary knowledge and technology to achieve regeneration of damaged tissues or organs. Regenerative therapy integrates knowledge and technology from multiple disciplines, aiming to regenerate damaged tissues or organs ${ }^{12,13}$. The number of publications and RRI in stem cells for cartilage regeneration have been on the rise, especially in the past 5 years, suggesting that the proportion of this field among all academic fields is increasing (Fig. 2A). In addition, more than half of the top 5 journals in this field have an impact factor of 5 or more (Fig. 4), which means that the topic of 
stem cells for cartilage regeneration has drawn widespread attention according to the method used to calculate the impact factor. Therefore, more in-depth studies will be published in the future and prove the feasibility and application prospects of stem cells for cartilage.

In terms of country and regional distribution, the total number of publications and citations of China ranks first in the world. However, in terms of the H-index, the USA still ranked first, indicating its great influence (Fig. 2B, Fig. 2D). When we further analyse the average citation rate (only compare countries with more than 100 publications), the average citation rate of China is 16.88 , while those of the United States and South Korea are 25.03 and 28.49, respectively (Table 1). Although China's contribution in this field is nonignorable, the centrality needs to be further improved. The influence and contribution of scientific institutions are an important part of that of a country or region. Nine of the top 10 institutions are located in China, and the top 10 institutions in China account for $70 \%$ of the total publication number. However, among the top 5 institutions in China in terms of publication volume, none of them rank among the top 5 in the world in terms of total citations (Table 2, Fig. 3). Although the citation rate of the article is not completely equal to the importance of academic achievements, it can still reflect some practical problems when a low citation rate becomes a common phenomenon. From the perspective of time distribution, the concentrated years of article output of China were approximately 2018 (Fig. 2D). However, there were no keywords or references with a sudden change in the number of citations in the past 3 years (Fig. 5D, Fig. 6D). This discrepancy suggests that scientific achievements in China have not formed a hot topic in this field. These results indicate that most academic achievements are the completion and supplementation of previous breakthrough results instead of original creative discoveries and techniques. The National Natural Science Foundation of China (NSFC) is one of the main channels for supporting basic research in China, and a series of changes in the NSFC application rules have taken place in recent years, aiming to improve the quality and innovativeness of publications. We believe that the centrality of China will gradually increase in the future.

As far as authors are concerned, SEKIYA I (Tokyo Medical \& Dental University, JAPAN) and YANG F (Stanford University, USA) are the most productive. However, in terms of the number of citations, the data of Toh WS (National University of Singapore, Singapore) are particularly attractive (Table 3). He has published 10 papers on stem cells for cartilage regeneration, but the total number of citations has reached 740 . The total citations of Toh WS accounted for approximately $60 \%$ of Singapore and $70 \%$ of National University of Singapore (Table 1, Table 2). In 2007, Toh WS successfully constructed an experimental system in which human embryoid body (EB)-derived cells could directly differentiate into chondrocytes under specific culture conditions ${ }^{14}$. In 2009, Toh WS and his team improved this highdensity micromass model system. Through using a more effective combination of growth factors and extracellular matrix substrate, they isolated a highly expandable and homogenous chondrogenic cell population named TC1, providing a potential source of chondrogenic cells for scientific research and clinical application ${ }^{15}$. In 2010, Toh WS and his team constructed hESC-derived chondrogenic cellengineered cartilage (HCCEC) from the abovementioned cell population in the culture of hyaluronic acid (HA)-based hydrogel and verified its long-term viability and safety in a rat model, providing a practical 
strategy of applying hESCs for cartilage regeneration ${ }^{4}$. In 2014, Toh WS and his team made a systematic description of the various tools and techniques of the culture system, tissue engineering protocol, and analytical methods, as well as the improvements ${ }^{16}$. Afterwards, Toh WS and his team shifted the focus of research to mesenchymal stem cell-derived exosomes and illustrated the underlying mechanisms of MSC exosomes in the biological behaviour of chondrocytes, extracellular matrix homeostasis, and immune reactivity in cartilage regeneration ${ }^{17}$. In addition, the author and his team published 3 reviews and summarized the potential and perspective of human embryonic stem cells (ESCs) in cartilage tissue engineering and regenerative medicine, the interactions between stem cells and extracellular matrix for cartilage regeneration, and the potential and perspective of MSC exosomes in cartilage regeneration. Among publications of Toh WS, 3 articles had more than 100 citations, and the topics of these 3 articles were "Cartilage repair using hyaluronan hydrogel-encapsulated human embryonic stem cell-derived chondrogenic cells" (DOI: 10.1016/j.biomaterials.2010.05.064, PMID: 20619789), "MSC exosomes as a cell-free MSC therapy for cartilage regeneration: implications for osteoarthritis treatment" (DOI: 10.1016/j.semcdb.2016.11.008, PMID: 27871993), and “MSC exosomes mediate cartilage repair by enhancing proliferation, attenuating apoptosis and modulating immune reactivity' (DOI:

10.1016/j.biomaterials.2017.11.028, PMID: 29182933).

As a key node in the co-citation network, Jo Ch's article (doi:10.1002/stem.1634) not only had the largest number of citations (Table 4) but also had the highest impact (Fig. 5A, Fig. 5B). Jo Ch is the first author, and the corresponding author of this article is Kang Sup Yoon. Kang Sup Yoon and his team substantiated the safety and efficacy of intra-articular injection of autologous AD-MSCs through clinical index, radiological evaluation, arthroscopic evaluation and histological evaluations. More importantly, Kang Sup Yoon and his team evaluated the effects of different doses of AD-MSCs injected into articular tissue. It is currently known that a sufficient number of MSCs can inhibit articular cartilage degradation and promote its regeneration ${ }^{18}$. However, when evaluating efficacy and safety comprehensively, the optimal cell dose needs to be clarified. Even if it is not the optimal dose, Kang Sup Yoon and his team suggested that at least $1.0 \times 10^{8} \mathrm{MSC}$ per injection would be a prerequisite for consistently good results, preliminarily exploring the therapeutic dose ${ }^{19}$. In conclusion, this proof-of-concept clinical trial confirmed the effectiveness of intra-articular injection of MSCs and gave a fair opinion on the injection dose, promising to encourage larger clinical research and application.

In the "Clinical study", the latest word was "double-blind" (AAY 2018.75), with 8 times (Fig. 6C), which heralds future trends in the biological study of stem cells for cartilage regeneration. A double-blind trial is a test where neither the tester nor the testees know the exact group of testees, which helps to avoid cognitive bias $^{20}$. Due to its higher cost, there are very few double-blind trials in this field (table 5). A double-blind trial from South Korea explored the effectiveness and safety of genetically engineered autologous chondrocytes, which were named TissueGene-C (TG-C), for knee osteoarthritis. TG-C was administered by a single intra-articular injection, and then subjective and objective assessments were performed by a clinical rating scale and radiographic evaluation ${ }^{21}$. Although subjective evaluation shows the effectiveness of this treatment, changes in joint space widths, bone area and cartilage thicknesses 
only show a trend that is not statistically significant. This research has laid the foundation for the development of double-blind trials in this field. On the basis of reality and possibility, double-blind experiments in stem cells for cartilage regeneration should also be attempted.

In tissue engineering, the latest word was "3D printing" (AAY 2019.625), with 9 times (Fig. 6C). 3D printing was proposed by Chuck Hull in 1983 and includes two main processes: computer-aided design (CAD) and $3 \mathrm{D}$ printing to form products. In the 2000s, 3D printing was used in the production of surgical models and later developed to be used in the production of live cell structures, including articular cartilage. Regarding the product formation process, there are also different strategies: inkjet printing, laser-assisted printing, and bioextrusion ${ }^{22,23}$. The materials used for 3D printing (bioink) are a hot and difficult point. Bioink mainly consists of two parts: biomaterials as scaffolds (2nd highest frequency keywords in tissue engineering clusters) and embedded cells ${ }^{24}$. Biomaterials used for tissue engineering need to have biocompatibility, biodegradability, and porosity, and they are mainly divided into natural polymers (alginate, gelatine, chitosan, and hyaluronan) or synthetic polymers (PCL, PGA, PEG) at present ${ }^{25}$. After being modified, natural polymers can be made into a more stable form, hydrogels, which have been widely used in 3D printing ${ }^{26}$. Synthetic polymers could be combined or coated with hydrogels to enhance their biocompatibility. Biomaterials can play a variety of roles depending on the embedded cell types, including chondrogenic differentiation (highest frequency keywords in tissue engineering clusters).

As mentioned earlier, chondrocytes have limited expansion efficiency and potential for dedifferentiation in vivo. In contrast, the application of stem cells in tissue engineering is more promising. According to current research, the mechanism underlying cartilage regeneration in stem cells is mainly attributed to direct differentiation of chondrocytes and paracrine secretion ${ }^{27,28}$. BM-MSCs are one of the most commonly used cell types in tissue engineering (table 5), and they can be induced into multiple cell types, including chondrocytes. AD-MSCs are easily accessible via minimally invasive procedures, and they have the characteristics of synthesizing more collagen than other MSC types. SF-MSCs, which can be isolated from synovial fluid and synovial membrane, have higher chondrogenic potential among osteoarticular cell types. Dental pulp MSCs have already been used in cartilage tissue engineering and have shown potential for hyaline-like cartilage formation with the synthesis of ECM components, such as aggrecan or collagen ${ }^{29,30}$. In addition to biological materials and stem cell types, environmental conditions and differentiation-promoting factors are also key points for 3D printing technology. For example, the surrounding medium's oxygen content affects the chondrogenic differentiation of MSCs ${ }^{31,32}$, and TGF- $\beta 1$ can promote and stabilize the chondrogenic phenotype ${ }^{33}$. Overall, 3D printing is a microcosm of tissue engineering. Whether it can be promoted clinically depends on many factors, and 3D printing is an important direction of future research.

In the biological study, the latest word was "extracellular vesicles" (AAY 2018.9091), with 12 times (Fig. 6C). Extracellular vesicles are released from the cell surface to body fluid, and they can transfer multiple types of cargo, such as lipids, nucleic acids and proteins, contributing to intercellular communication ${ }^{34}$. Exosomes, microvesicles (MVs) and apoptotic bodies are currently the most attractive 
categories of extracellular vesicles, and they are different in generation mechanism and size ${ }^{35}$. According to publication numbers, exosomes have attracted more attention among researchers. Currently, exosomes have been identified from multiple MSCs, including those derived from AD-MSCs, BM-MSCs, US-MSCs, hESCs, and $\mathrm{iPSCs}{ }^{36,37}$. When MSCs sense changes in the surrounding microenvironment, they can secrete exosomes to interact with other cells. Exosomes have been proven to regulate the biological behaviour of chondrocytes, to modulate immune responses and to restore homeostasis to the ECM, thereby promoting cartilage regeneration ${ }^{38,39}$. Although the therapeutic efficacy, biosafety, kinetics and biodistribution of MSC exosomes need to be studied in depth, the regenerative potency of MSC exosomes provides new perspectives for the development of a cell-free MSC therapy strategy for cartilage regeneration.

The purpose of clinical trials is to repair damaged articular cartilage. Without undergoing such a major operation as joint replacement, the application of stem cells is expected to replace traditional medical treatment and achieve the goal of radical cure. According to current clinical trials, except for CARTISTEM, which uses commercial hUCB-MSCs ${ }^{40}$, all other clinical trials use autologous stem cells. The entire process includes collection, in vitro expansion, and intra-articular injection ${ }^{41}$. Subjective clinical scores, imaging evidence, and histological evaluation are currently the most important evaluation methods. The effectiveness, feasibility and safety of the concept of using mesenchymal stem cells in cartilage repair have been verified (Table 5). However, there are still some issues that need to be resolved. First, the most suitable cell source needs further exploration. Except for CARTISTEM, all other clinical trials use autologous stem cells, and the most commonly used type is BM-MSCs. The horizontal comparison of different types of cells is still missing. Second, it is unclear whether MSCs mainly differentiate into chondrocytes directly or more through paracrine forms to promote cartilage formation. Third, the optimal therapeutic course and dose need to be further explored. NCT01207661 indicated that the effect of a single injection was improved in the first 12 months and decreased from the 12 th month ${ }^{42}$. NCT01873625 also explored the number of cells per injection and provided a reference value of 40 million ${ }^{43}$. However, the best course of treatment and therapeutic dose need more exploration. In addition, current clinical trials are mainly short-term follow-ups, typically only 24 months or less. Long-term efficacy and safety evaluations need further verification.

This study investigated publications on stem cells for cartilage regeneration extracted from the Web of Science database and discussed hot issues in this comprehensive and objective field. However, limitations are inevitable. First, we only enrolled publications in the language of English, which may miss some important non-English studies in this field. In addition, although keywords can reflect the subject of the article to a certain extent, the preference of different authors when using keywords and the shortness of keywords will lead to bias in trend analysis.

\section{Conclusion}

Overall, this study summarized and analysed the global research trends concerning stem cells for cartilage regeneration. The annual output of related publications has grown remarkably, and stem cells

Page $11 / 24$ 
for cartilage regeneration have positive development prospects. China and the USA have made the largest contributions in this field. 3D printing, extracellular vesicles, and double-blind clinical trials are research hotspots and are likely to be promising in the near future. Clinical trials with larger sample sizes and longer follow-up periods are needed for clinical transformation.

\section{Declarations}

\section{Acknowledgements}

Not applicable.

\section{Authors' contributions}

Shuogui Xu and Panyu Zhou were responsible for the study design. Jianghong Wu and Kaiwen Wu were responsible for data collection. Sheng Wang and Zhentao Zhang were responsible for analyzing the data. Demeng Xia and Jianghong Wu were responsible for manuscript drafting. All authors have critically reviewed and approved the final manuscript.

\section{Funding}

The National Science Foundation for Young Scientists of China (No. 81601910), the Shanghai Rising-Star Program (No. 18QA1405400), the Military Medical Research Foundation from the Naval Military Medical University (No. 2017JS15), and the China Postdoctoral Science Foundation Grant (No. 2016M592936), Incubation project of military medical science and Technology Youth cultivation plan (20QNPY036).

\section{Consent for publication}

Not applicable.

\section{Availability of data and materials}

The data that support the findings of this study are available from Web of Science (https://www.webofknowledge.com), which are all publicly available.

\section{Ethics approval and consent to participate}

This study does not involve animal and/or human tissue/individual data/participants, there is no ethics related issues. No permissions were required touse any repository data involved in the present study.

\section{Competing interests}

The authors declare that they have no competing interests.

\section{References}


1. Carballo, C., Nakagawa, Y., Sekiya, I. \& Rodeo, S.J.C.i.s.m. Basic Science of Articular Cartilage. 36, 413-425 (2017).

2. Le, H., et al. Mesenchymal stem cells for cartilage regeneration. 11, 2041731420943839 (2020).

3. Murphy, M., et al. Articular cartilage regeneration by activated skeletal stem cells. 26, 1583-1592 (2020).

4. Toh, W.S., et al. Cartilage repair using hyaluronan hydrogel-encapsulated human embryonic stem cell-derived chondrogenic cells. Biomaterials 31, 6968-6980 (2010).

5. Zellner, J., et al. Clinical and Radiological Regeneration of Large and Deep Osteochondral Defects of the Knee by Bone Augmentation Combined With Matrix-Guided Autologous Chondrocyte Transplantation. 45, 3069-3080 (2017).

6. Toh, W.S., Lee, E.H. \& Cao, T. Potential of human embryonic stem cells in cartilage tissue engineering and regenerative medicine. Stem cell reviews and reports 7, 544-559 (2011).

7. Li, J., et al. Cartilage regeneration using arthroscopic flushing fluid-derived mesenchymal stem cells encapsulated in a one-step rapid cross-linked hydrogel. 79, 202-215 (2018).

8. Wang, K., et al. Chondrogenic Progenitor Cells Exhibit Superiority Over Mesenchymal Stem Cells and Chondrocytes in Platelet-Rich Plasma Scaffold-Based Cartilage Regeneration. 47, 2200-2215 (2019).

9. Wells, K., et al. Cellular and Clinical Analyses of Autologous Bone Marrow Aspirate Injectate for Knee Osteoarthritis: A Pilot Study. (2020).

10. Baldiotti, A., et al. The Top 100 Most-Cited Papers in Cariology: A Bibliometric Analysis. 1-9 (2020).

11. Yan, W., et al. Bibliometric evaluation of 2000-2019 publications on functional near-infrared spectroscopy. 220, 117121 (2020).

12. El-Jawhari, J., et al. The CD45lowCD271high Cell Prevalence in Bone Marrow Samples May Provide a Useful Measurement of the Bone Marrow Quality for Cartilage and Bone Regenerative Therapy. 99, 1305-1313 (2017).

13. Zhang, W., Ouyang, H., Dass, C. \& Xu, J.J.B.r. Current research on pharmacologic and regenerative therapies for osteoarthritis. 4, 15040 (2016).

14. Toh, W.S., Yang, Z., Heng, B.C. \& Cao, T. Differentiation of human embryonic stem cells toward the chondrogenic lineage. Methods in molecular biology (Clifton, N.J.) 407, 333-349 (2007).

15. Toh, W.S., et al. Differentiation and enrichment of expandable chondrogenic cells from human embryonic stem cells in vitro. Journal of cellular and molecular medicine 13, 3570-3590 (2009).

16. Toh, W.S. \& Cao, T. Derivation of Chondrogenic Cells from Human Embryonic Stem Cells for Cartilage Tissue Engineering. Methods in molecular biology (Clifton, N.J.) (2014).

17. Toh, W.S., Lai, R.C., Hui, J.H.P. \& Lim, S.K. MSC exosome as a cell-free MSC therapy for cartilage regeneration: Implications for osteoarthritis treatment. Seminars in cell \& developmental biology 67, 56-64 (2017).

18. Ichiseki, T., et al. Intraarticularly-Injected Mesenchymal Stem Cells Stimulate Anti-Inflammatory Molecules and Inhibit Pain Related Protein and Chondrolytic Enzymes in a Monoiodoacetate-Induced 
Rat Arthritis Model. 19(2018).

19. Jo, C., et al. Intra-articular injection of mesenchymal stem cells for the treatment of osteoarthritis of the knee: a proof-of-concept clinical trial. 32, 1254-1266 (2014).

20. Cho, S., et al. A Randomized, Double-Blind Trial Comparing the Pharmacokinetics of CT-P16, a Candidate Bevacizumab Biosimilar, with its Reference Product in Healthy Adult Males. 33, 173-181 (2019).

21. Kim, M., et al. A Multicenter, Double-Blind, Phase III Clinical Trial to Evaluate the Efficacy and Safety of a Cell and Gene Therapy in Knee Osteoarthritis Patients. 29, 48-59 (2018).

22. Wu, L., et al. Stiffness memory of indirectly 3D-printed elastomer nanohybrid regulates chondrogenesis and osteogenesis of human mesenchymal stem cells. 186, 64-79 (2018).

23. Messaoudi, O., et al. Stem Cells and Extrusion 3D Printing for Hyaline Cartilage Engineering. 10(2020).

24. Kim, M., et al. Decellularized extracellular matrix-based bio-ink with enhanced 3D printability and mechanical properties. 12, 025003 (2020).

25. Shen, S., et al. Three Dimensional Printing-Based Strategies for Functional Cartilage Regeneration. 25, 187-201 (2019).

26. Diloksumpan, P., et al. Combining multi-scale 3D printing technologies to engineer reinforced hydrogel-ceramic interfaces. 12, 025014 (2020).

27. Iturriaga, L., Hernáez-Moya, R., Erezuma, I., Dolatshahi-Pirouz, A. \& Orive, G.J.E.o.o.b.t. Advances in stem cell therapy for cartilage regeneration in osteoarthritis. 18, 883-896 (2018).

28. Kim, Y., Choi, J. \& Kim, K.J.B.j. Mesenchymal Stem Cell-Derived Exosomes for Effective Cartilage Tissue Repair and Treatment of Osteoarthritis. 15, e2000082 (2020).

29. Kabir, W., Di Bella, C., Jo, I., Gould, D. \& Choong, P.J.T.e.P.B., Reviews. In VivoHuman Stem Cell Based Tissue Engineering for Cartilage Repair: A Systematic Review. (2020).

30. Tan, A. \& Hung, C.J.S.c.t.m. Concise Review: Mesenchymal Stem Cells for Functional Cartilage Tissue Engineering: Taking Cues from Chondrocyte-Based Constructs. 6, 1295-1303 (2017).

31. Anderson, D., et al. Responses to altered oxygen tension are distinct between human stem cells of high and low chondrogenic capacity. 7, 154 (2016).

32. Portron, S., et al. Inverse regulation of early and late chondrogenic differentiation by oxygen tension provides cues for stem cell-based cartilage tissue engineering. 35, 841-857 (2015).

33. Futrega, K., Robey, P., Klein, T., Crawford, R. \& Doran, M.J.C.b. A single day of TGF- $\beta 1$ exposure activates chondrogenic and hypertrophic differentiation pathways in bone marrow-derived stromal cells. 4, 29 (2021).

34. Allan, D., Tieu, A., Lalu, M. \& Burger, D.J.S.c.t.m. Mesenchymal stromal cell-derived extracellular vesicles for regenerative therapy and immune modulation: Progress and challenges toward clinical application. 9, 39-46 (2020). 
35. Lawson, C., Vicencio, J., Yellon, D. \& Davidson, S.J.T.J.o.e. Microvesicles and exosomes: new players in metabolic and cardiovascular disease. 228, R57-71 (2016).

36. Zhang, S., et al. MSC exosomes mediate cartilage repair by enhancing proliferation, attenuating apoptosis and modulating immune reactivity. Biomaterials 156, 16-27 (2018).

37. $\mathrm{Xu}, \mathrm{X}$., et al. Exosome-mediated delivery of kartogenin for chondrogenesis of synovial fluid-derived mesenchymal stem cells and cartilage regeneration. 120539 (2020).

38. Tan, S., et al. In VivoMesenchymal Stem Cell Exosomes for Cartilage Regeneration: A Systematic Review of Preclinical Studies. (2020).

39. Zhang, S., et al. MSC exosomes mediate cartilage repair by enhancing proliferation, attenuating apoptosis and modulating immune reactivity. 156, 16-27 (2018).

40. Park, Y., Ha, C., Lee, C., Yoon, Y. \& Park, Y.J.S.c.t.m. Cartilage Regeneration in Osteoarthritic Patients by a Composite of Allogeneic Umbilical Cord Blood-Derived Mesenchymal Stem Cells and Hyaluronate Hydrogel: Results from a Clinical Trial for Safety and Proof-of-Concept with 7 Years of Extended Follow-Up. 6, 613-621 (2017).

41. Al-Najar, M., et al. Intra-articular injection of expanded autologous bone marrow mesenchymal cells in moderate and severe knee osteoarthritis is safe: a phase I/II study. 12, 190 (2017).

42. Emadedin, M., et al. Long-Term Follow-up of Intra-articular Injection of Autologous Mesenchymal Stem Cells in Patients with Knee, Ankle, or Hip Osteoarthritis. 18, 336-344 (2015).

43. Shadmanfar, S., et al. Intra-articular knee implantation of autologous bone marrow-derived mesenchymal stromal cells in rheumatoid arthritis patients with knee involvement: Results of a randomized, triple-blind, placebo-controlled phase 1/2 clinical trial. 20, 499-506 (2018).

\section{Tables}

Table 1. The top 20 countries that contributed publications on stem cells for cartilage regeneration. 


\begin{tabular}{|lllll|}
\hline Country & No. of Publications & Sum of Citations & Citations & H-index \\
\hline PEOPLES R CHINA & 317 & 5351 & 4993 & 37 \\
\hline USA & 203 & 5081 & 4903 & 39 \\
\hline SOUTH KOREA & 107 & 3048 & 2938 & 29 \\
\hline JAPAN & 82 & 1374 & 1313 & 21 \\
\hline GERMANY & 59 & 1054 & 1041 & 18 \\
\hline ENGLAND & 54 & 974 & 949 & 17 \\
\hline ITALY & 45 & 1244 & 1223 & 18 \\
\hline NETHERLANDS & 37 & 668 & 652 & 16 \\
\hline TAIWAN & 35 & 467 & 457 & 11 \\
\hline IRAN & 30 & 493 & 478 & 11 \\
\hline SPAIN & 29 & 974 & 960 & 13 \\
\hline AUSTRALIA & 25 & 304 & 294 & 9 \\
\hline SINGAPORE & 25 & 1253 & 1216 & 15 \\
\hline SWITZERLAND & 21 & 375 & 372 & 13 \\
\hline MALAYSIA & 20 & 577 & 562 & 9 \\
\hline FRANCE & 19 & 463 & 461 & 10 \\
\hline IRELAND & 19 & 585 & 575 & 12 \\
\hline INDIA & 18 & 360 & 229 & 8 \\
\hline BRAZIL & 13 & 109 & 7 & 77 \\
\hline CANADA & 12 & & & 107 \\
\hline
\end{tabular}

Table 2. Top 20 institutions with the most publications in the field of stem cells for cartilage regeneration. 


\begin{tabular}{|llll|}
\hline Institution & Country & $\begin{array}{l}\text { No. of } \\
\text { Publications }\end{array}$ & $\begin{array}{l}\text { No. of } \\
\text { Citations }\end{array}$ \\
\hline SHANGHAI JIAO TONG UNIVERSITY & CHINA & 34 & 4120 \\
\hline PEKING UNIVERSITY & CHINA & 27 & 285 \\
\hline SICHUAN UNIVERSITY & CHINA & 26 & 452 \\
\hline $\begin{array}{l}\text { CHINESE PEOPLE S LIBERATION ARMY GENERAL } \\
\text { HOSPITAL }\end{array}$ & CHINA & 22 & 445 \\
\hline CHINESE ACADEMY OF SCIENCES & CHINA & 21 & 218 \\
\hline NATIONAL UNIVERSITY OF SINGAPORE & SINGAPORE & 21 & 1051 \\
\hline CHINESE UNIVERSITY OF HONG KONG & CHINA & 20 & 406 \\
\hline ZHEJIANG UNIVERSITY & CHINA & 20 & 478 \\
\hline SUN YAT SEN UNIVERSITY & CHINA & 18 & 490 \\
\hline NANJING MEDICAL UNIVERSITY & CHINA & 17 & 132 \\
\hline PENNSYLVANIA COMMONWEALTH SYSTEM OF & USA & 17 & 463 \\
\hline HIGHER EDUCATION PCSHE & & & 824 \\
\hline TOKYO MEDICAL DENTAL UNIVERSITY TMDU & JAPAN & 16 & 480 \\
\hline UNIVERSITY OF TWENTE & NETHERLANDS & 16 & 399 \\
\hline CENTRAL SOUTH UNIVERSITY & CHINA & 15 & 121 \\
\hline KYOTO UNIVERSITY & JAPAN & 15 & 257 \\
\hline FUDAN UNIVERSITY & CHINA & 14 & 181 \\
\hline HARVARD UNIVERSITY & USA & 14 & 458 \\
\hline INSTITUT NATIONAL DE LA SANTE ET DE LA & FRANCE & 14 & \\
\hline RECHERCHE MEDICALE INSERM & & 14 & \\
\hline UNIVERSITY OF PITTSBURGH & USA & \\
\hline WEST VIRGINIA UNIVERSITY & & 14 & \\
\hline
\end{tabular}

Table 3. Top 10 authors in the field of stem cells for cartilage regeneration ranked by publication number. 


\begin{tabular}{|lllll|}
\hline Author & Country & Affiliation & $\begin{array}{l}\text { No. of } \\
\text { Publications }\end{array}$ & $\begin{array}{l}\text { No. of } \\
\text { Citations }\end{array}$ \\
\hline SEKIYA I & JAPAN & Tokyo Medical \& Dental University & 14 & 387 \\
\hline YANG F & USA & Stanford University & 14 & 52 \\
\hline GUO QY & $\begin{array}{l}\text { PEOPLES R } \\
\text { CHINA }\end{array}$ & $\begin{array}{l}\text { Chinese People's Liberation Army } \\
\text { General Hospital }\end{array}$ & 13 & 229 \\
\hline PEI M & USA & West Virginia University & 13 & 276 \\
\hline PENG J & $\begin{array}{l}\text { PEOPLES R } \\
\text { CHINA }\end{array}$ & $\begin{array}{l}\text { Chinese People's Liberation Army } \\
\text { General Hospital }\end{array}$ & 12 & 191 \\
\hline ZHANG X & $\begin{array}{l}\text { PEOPLES R } \\
\text { CHINA }\end{array}$ & Peking University & 11 & 146 \\
\hline KOH YG & $\begin{array}{l}\text { SOUTH } \\
\text { KOREA }\end{array}$ & Yonsei Sarang Hosp & 10 & 240 \\
\hline TOH WS & SINGAPORE & National University of Singapore & 10 & 740 \\
\hline $\begin{array}{l}\text { MOBASHERI } \\
\text { A }\end{array}$ & FINLAND & University of Oulu & 10 & 502 \\
\hline TUAN RS & $\begin{array}{l}\text { PEOPLES R } \\
\text { CHINA }\end{array}$ & Chinese University of Hong Kong & 10 & 342 \\
\hline
\end{tabular}

Table 4. Top 10 references with the most citations in the field of stem cells for cartilage regeneration. 


\begin{tabular}{|c|c|c|c|c|c|}
\hline Title & $\begin{array}{l}\text { Corresponding } \\
\text { author }\end{array}$ & Journal & IF & $\begin{array}{l}\text { Publication } \\
\text { Year }\end{array}$ & $\begin{array}{l}\text { Total } \\
\text { Citations }\end{array}$ \\
\hline $\begin{array}{l}\text { Intra-Articular Injection } \\
\text { of Mesenchymal Stem } \\
\text { Cells for the Treatment } \\
\text { of Osteoarthritis of the } \\
\text { Knee: A Proof-of- } \\
\text { Concept Clinical Trial }\end{array}$ & Yoon, KS & STEM CELLS & 6.523 & 2014 & 424 \\
\hline $\begin{array}{l}\text { Isolation of adipose- } \\
\text { derived stem cells and } \\
\text { their induction to a } \\
\text { chondrogenic } \\
\text { phenotype }\end{array}$ & Guilak, F & $\begin{array}{l}\text { NATURE } \\
\text { PROTOCOLS }\end{array}$ & 8.326 & 2010 & 291 \\
\hline $\begin{array}{l}\text { Chitosan, hyaluronan } \\
\text { and chondroitin sulfate } \\
\text { in tissue engineering } \\
\text { for cartilage } \\
\text { regeneration: A review }\end{array}$ & $\begin{array}{l}\text { Muzzarelli, } \\
\text { RAA }\end{array}$ & $\begin{array}{l}\text { CARBOHYDRATE } \\
\text { POLYMERS }\end{array}$ & 3.479 & 2012 & 267 \\
\hline $\begin{array}{l}\text { Treatment of Knee } \\
\text { Osteoarthritis With } \\
\text { Allogeneic Bone } \\
\text { Marrow Mesenchymal } \\
\text { Stem Cells: A } \\
\text { Randomized Controlled } \\
\text { Trial }\end{array}$ & $\begin{array}{l}\text { Garcia- } \\
\text { Sancho, J }\end{array}$ & TRANSPLANTATION & 3.69 & 2015 & 258 \\
\hline $\begin{array}{l}\text { Treatment of Knee } \\
\text { Osteoarthritis With } \\
\text { Autologous } \\
\text { Mesenchymal Stem } \\
\text { Cells: A Pilot Study }\end{array}$ & $\begin{array}{l}\text { Garcia- } \\
\text { Sancho, J }\end{array}$ & TRANSPLANTATION & 3.535 & 2013 & 251 \\
\hline $\begin{array}{l}\text { Infrapatellar fat pad- } \\
\text { derived mesenchymal } \\
\text { stem cell therapy for } \\
\text { knee osteoarthritis }\end{array}$ & Choi, YJ & KNEE & 3.781 & 2012 & 232 \\
\hline $\begin{array}{l}\text { Exosomes derived } \\
\text { from human } \\
\text { embryonic } \\
\text { mesenchymal stem } \\
\text { cells promote } \\
\text { osteochondral } \\
\text { regeneration }\end{array}$ & Toh, WS & $\begin{array}{l}\text { OSTEOARTHRITIS } \\
\text { AND CARTILAGE }\end{array}$ & 4.742 & 2016 & 230 \\
\hline $\begin{array}{l}\text { Hydrogels that mimic } \\
\text { developmentally } \\
\text { relevant matrix and N- } \\
\text { cadherin interactions } \\
\text { enhance MSC } \\
\text { chondrogenesis }\end{array}$ & Burdick, JA & $\begin{array}{l}\text { PROCEEDINGS OF } \\
\text { THE NATIONAL } \\
\text { ACADEMY OF } \\
\text { SCIENCES OF THE } \\
\text { UNITED STATES OF } \\
\text { AMERICA }\end{array}$ & 9.809 & 2013 & 223 \\
\hline $\begin{array}{l}\text { Exosomes derived } \\
\text { from miR-140-5p- } \\
\text { overexpressing human }\end{array}$ & Zhang, CQ & THERANOSTICS & 8.063 & 2017 & 197 \\
\hline
\end{tabular}


synovial mesenchymal

stem cells enhance

cartilage tissue

regeneration and

prevent osteoarthritis

of the knee in a rat

model

Comparative

evaluation of MSCs

Zhang, CQ

BIOMATERIALS

$7.604 \quad 2012$

179

from bone marrow and

adipose tissue seeded

in PRP-derived scaffold

for cartilage

regeneration

Due to technical limitations, table 5 PDF is only available as a download in the Supplemental Files section.

\section{Figures}

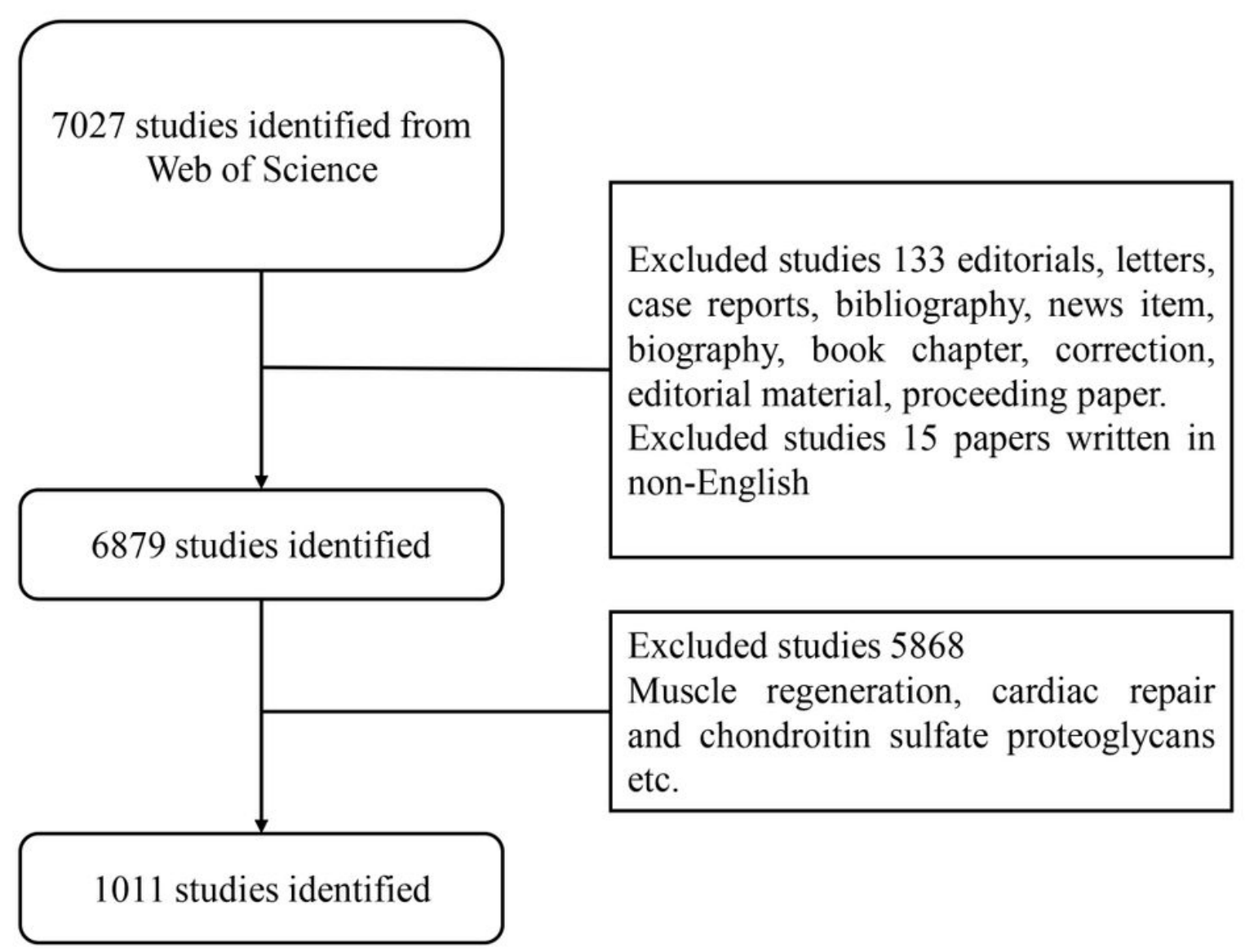

Figure 1 
Flow chart of the screening process for research on stem cells for cartilage.
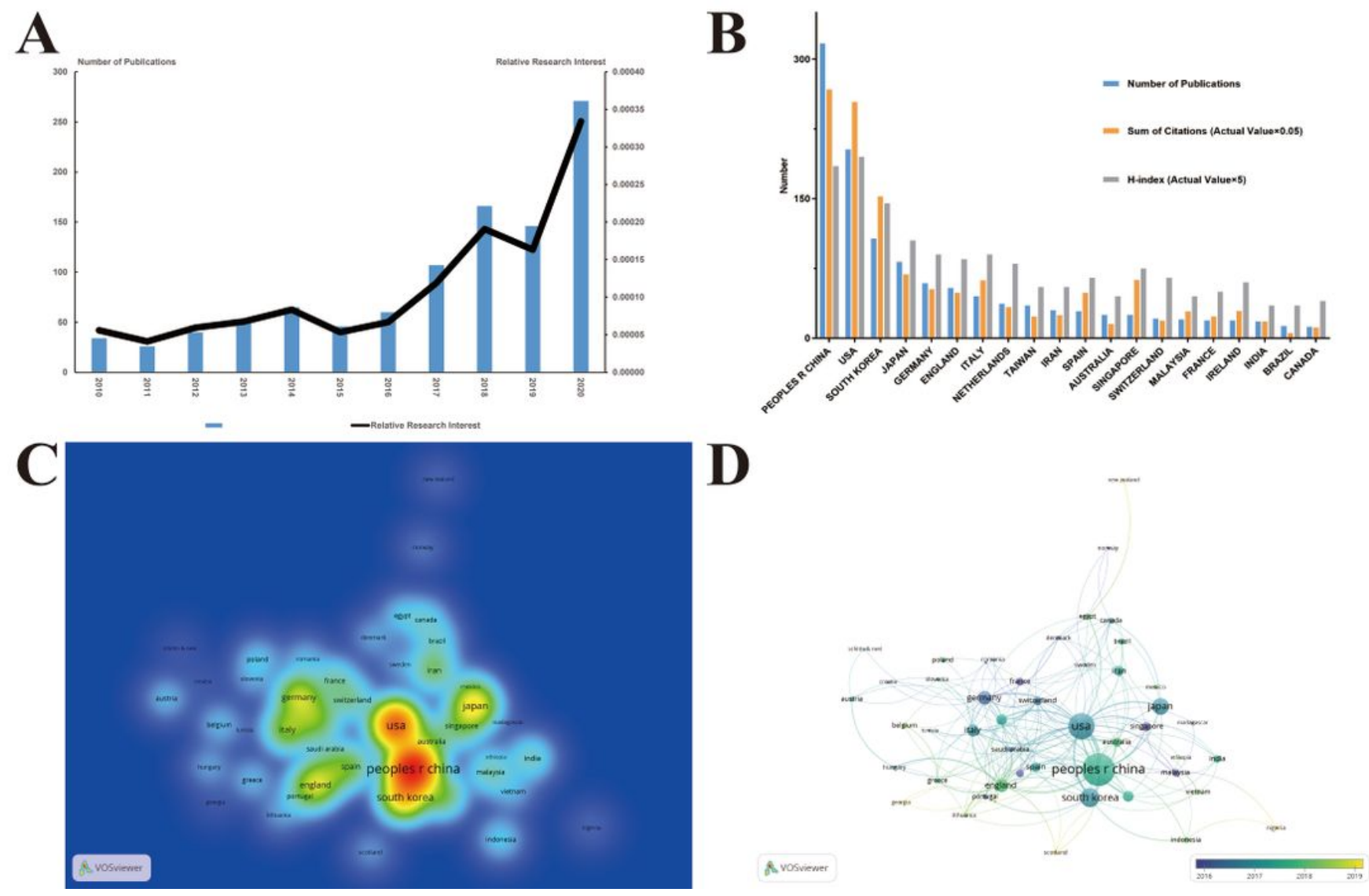

\section{Figure 2}

Articles related to stem cells for cartilage regeneration published worldwide. A. The annual publications worldwide and the relative research interest (RRI) for stem cells for cartilage regeneration. $B$. The number of publications, citation frequency (actual value $\times 0.05$ ), and $\mathrm{H}$-index (actual value $\times 5$ ) in the top 20 countries or regions. C. Density map of regional distribution of articles based on VOSviewer. D. Collaboration between countries or regions based on VOSviewer.

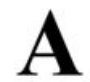

A voswewer

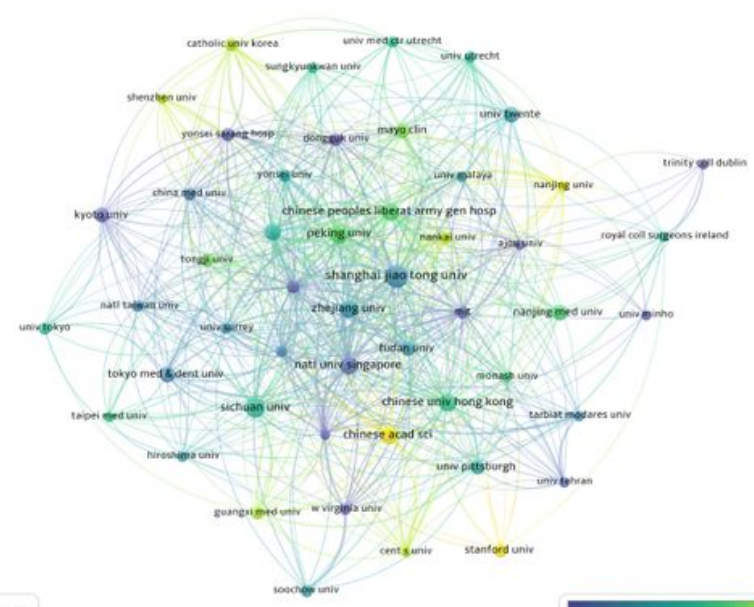

B

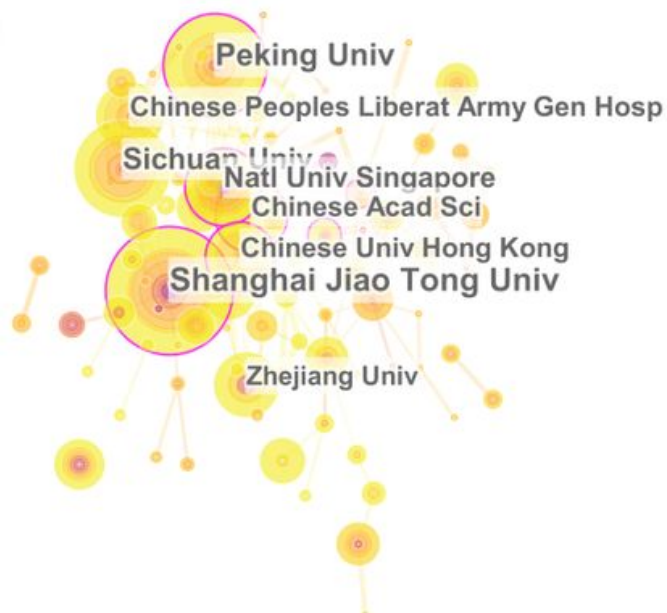




\section{Figure 3}

Articles on stem cells for cartilage regeneration published at different institutions. A. Collaboration between institutions based on VOSviewer; B. cooperation network analysis of institutions based on CiteSpace.
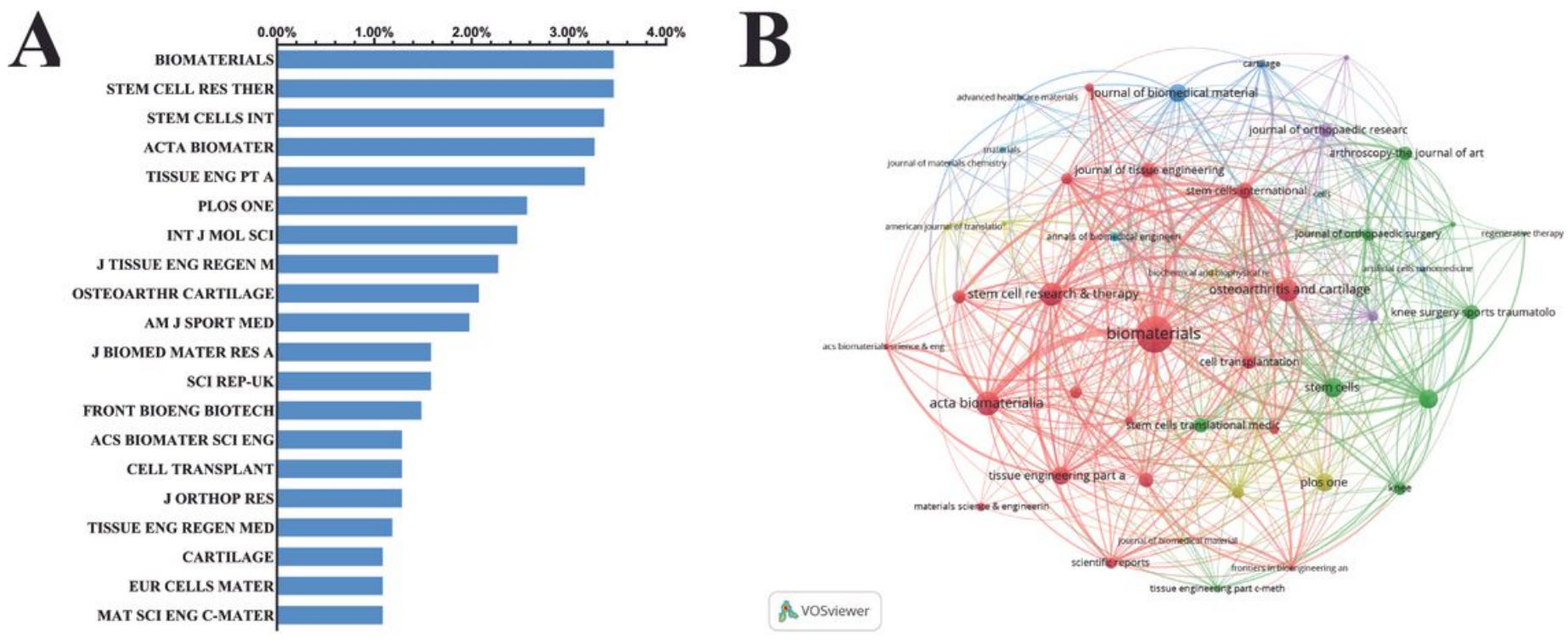

\section{Figure 4}

Articles published in different journals on stem cells for cartilage regeneration. A. Top 20 journals that produced the largest number of articles. B. Co-citation analysis of journals based on VOSviewer. 
$\mathbf{A}$

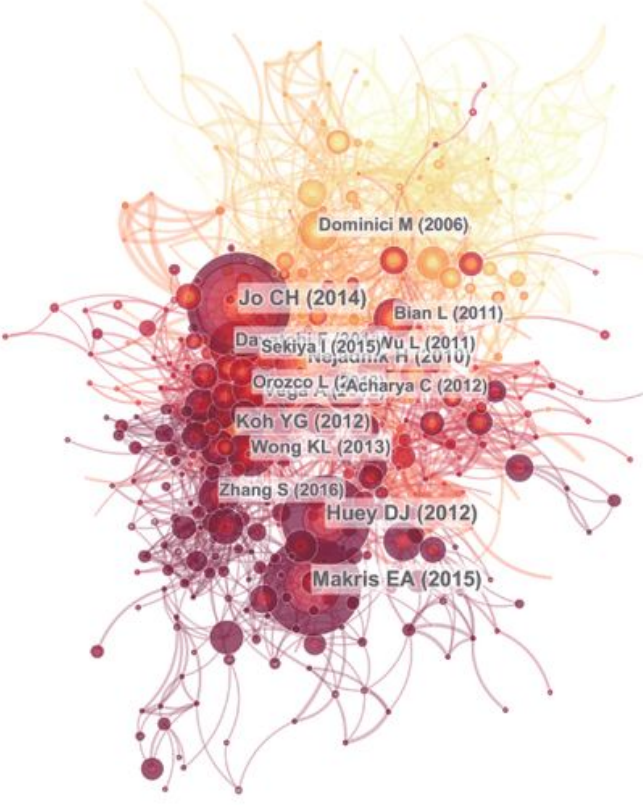

$\mathbf{C}$

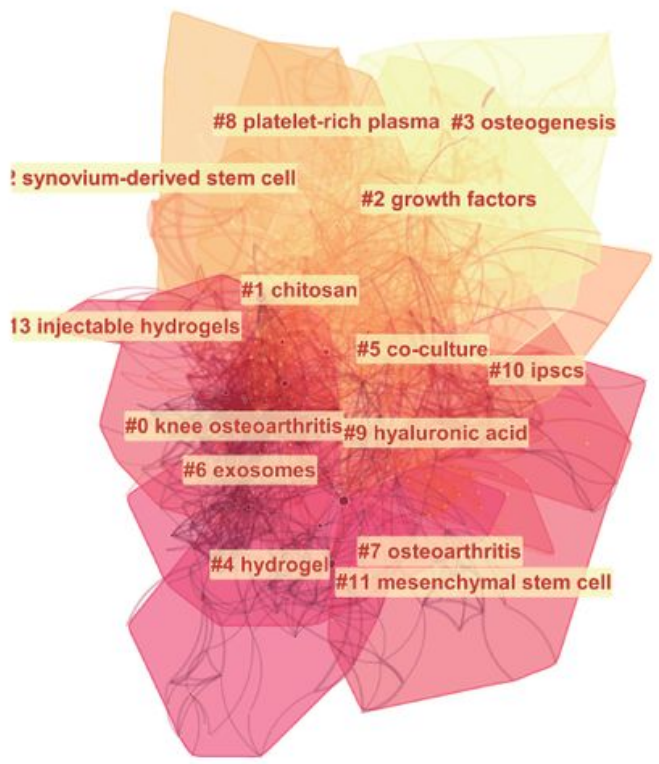

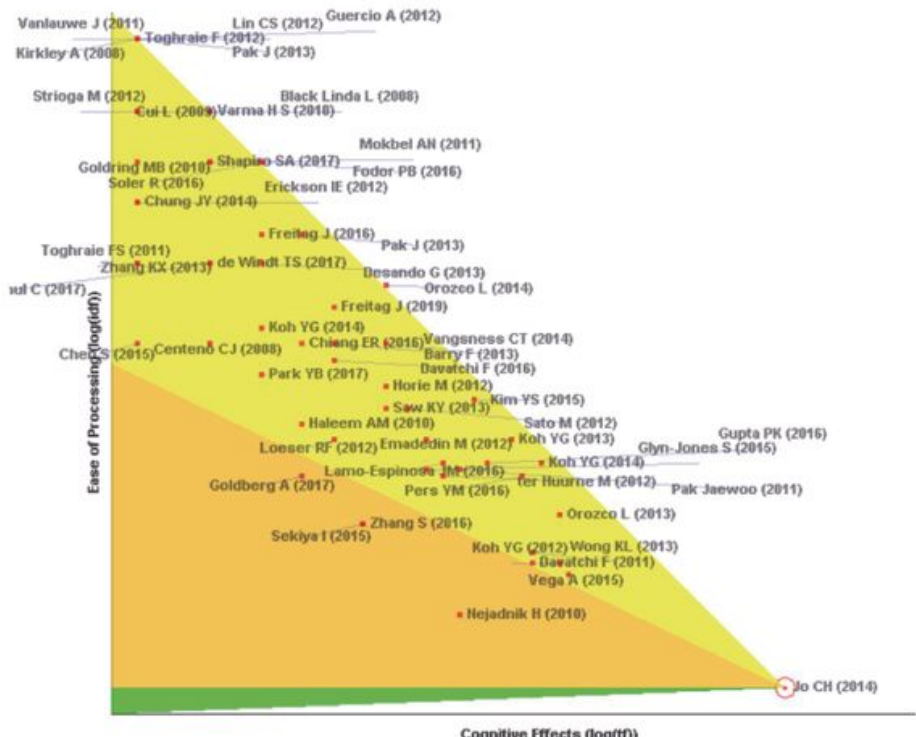

Top 25 References with the Strongest Citation Bursts

$\begin{array}{r}\begin{array}{r}\text { References } \\ \text { Engler AJ, 2006, CELL, V126, P677, DOI }\end{array} \\ \hline\end{array}$

Gimble JM, 2007, CIRC RES, V100, P1249, DOI

Hennig T, 2007, J CELL PHYSIOL, V211, P682, DO

De Bari C, 2004, ARTHRITIS RHEUM-US, V50, P142, DOI

Afizah H, 2007, TISSUE ENG, V13, P659, DOI

Lee KBL, 2007, STEM CELLS, V25, P2964, DOI

Dominici M, 2006, CYTOTHERAPY, V8, P315, DOI

Awad HA, 2004, BIOMATERIALS, V25, P3211, DOI

Pelttari K, 2006, ARTHRITIS RHEUM-US, V54, P3254, DOI

Sekiya I, 2005, CELL TISSUE RES, V320, P269, DOI

Dorotka R, 2005, BIOMATERIALS, V26, P3617, DOI

Toh WS, 2007, STEM CELLS, V25, P950, DOI

Koga H, 2008, CELL TISSUE RES, V333, P207, DOI

Indrawattana N, 2004, BIOCHEM BIOPH RES CO, V320, P914

Sakaguchi Y, 2005, ARTHRITIS RHEUM-US, V52, P2521, DOI

Chung C, 2008, ADV DRUG DELIVER REV, V60, P243, DO

Pelttari K, 2008, INJURY, V39, P0, DOI

Estes BT, 2006, ARTHRITIS RHEUM-US, V54, P1222, DOI

Steadman JR, 2003, ARTHROSCOPY, V19, P477, DOI

Koga $H, 2008$, ARTHRITIS RES THER, V10, PO, DOI

Goldring MB, 2006, J CELL BIOCHEM, V97, P33, DOI

Cheng NC, 2009, TISSUE ENG PT A, V15, P231, DOI

Knutsen G, 2004, J BONE JOINT SURG AM, V86A, P455, DOI

Mauck RL, 2006, OSTEOARTHR CARTILAGE, V14, P179, DOI

Li WJ, 2005, BIOMATERIALS, V26, P599, DOI

\begin{tabular}{|c|c|c|c|}
\hline ea & Strength Be & & \\
\hline 2006 & 4.00722010 & 2012 & \\
\hline 2007 & 3.13582010 & $2013=$ & \\
\hline 2007 & 5.28572010 & 2014 & \\
\hline 2004 & 2.5012010 & 2011 & \\
\hline 007 & 5.51632010 & 2015 & \\
\hline 07 & 6.70452010 & 2015 & \\
\hline 006 & 13.28782010 & 2014 & \\
\hline 2004 & 3.75382010 & 2011 & \\
\hline 2006 & 10.61052010 & 2014 & \\
\hline 2005 & 4.58112010 & 2012 & \\
\hline 2005 & 2.5012010 & 2011 & \\
\hline 2007 & 3.51952010 & 2014 & \\
\hline 08 & 5.91212010 & 2015 & \\
\hline 2004 & 2.5012010 & 2011 & \\
\hline 005 & 11.55532010 & 2013 & \\
\hline 08 & 7.49962010 & 201 & \\
\hline 2008 & 5.75792010 & 2013 & \\
\hline 2006 & 5.23282010 & 2013 & \\
\hline 2003 & 3.75382010 & 2011 & \\
\hline 2008 & 3.93542010 & $2015=$ & \\
\hline 006 & 7.49962010 & 2014 & \\
\hline 2009 & 3.14642010 & 2015 & \\
\hline 2004 & 4.58112010 & 2012 & \\
\hline 2006 & 4.4022010 & 2014 & \\
\hline 005 & 3.65582011 & 2012 & \\
\hline
\end{tabular}

\section{Figure 5}

Mapping of references in studies on stem cells for cartilage regeneration. A. A simplified co-citation network of references about stem cells for cartilage regeneration based on CiteSpace (burst references are represented by red nods). B. The article with the highest citation rate was also processed for in-depth analysis. C. Clustering analysis of the co-citation network based on CiteSpace. D. Top 25 references with strongest citation bursts based on CiteSpace. 

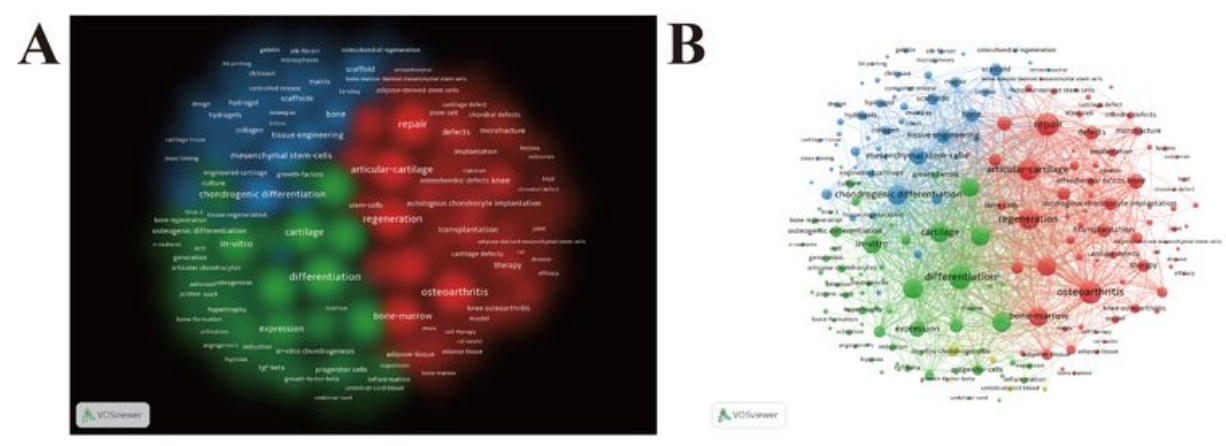

C

D Top 20 Keywords with the Strongest Citation Bursts

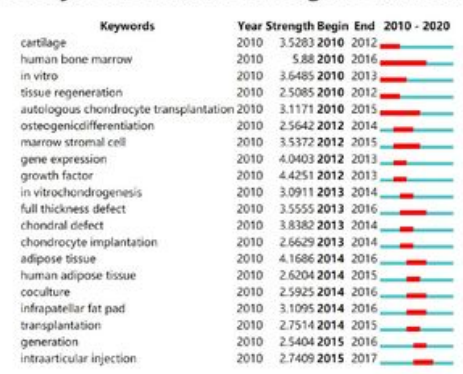

$\mathbf{E}$

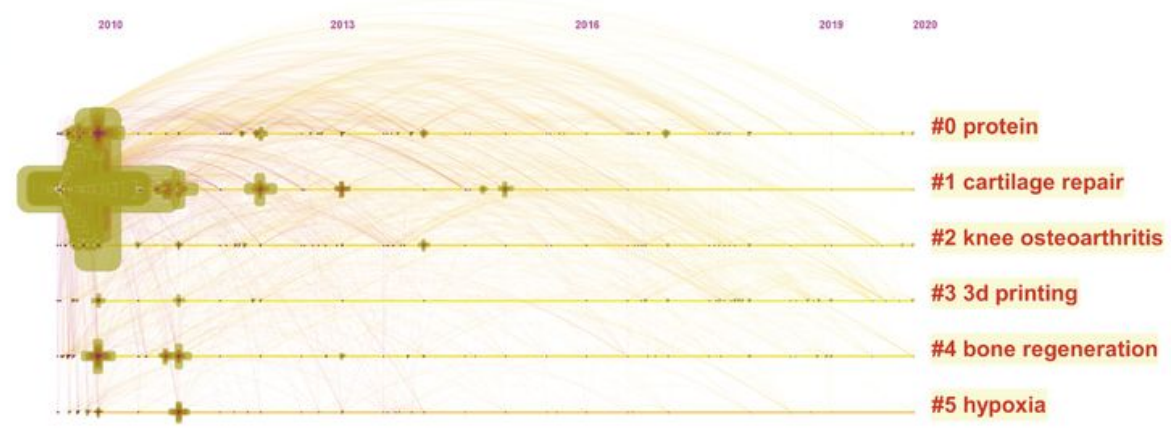

Figure 6

Mapping of keywords in studies on stem cells for cartilage regeneration. A. Clustering analysis of key words based on VOSviewer. B. Network visualization of keywords based on VOSviewer. C. Chronological order of keywords based on VOSviewer. D. Top 20 keywords with the strongest citation bursts based on CiteSpace. E. Keyword timeline visualization from 2010 to 2020.

\section{Supplementary Files}

This is a list of supplementary files associated with this preprint. Click to download.

- TableS1.docx

- Table5.pdf 